\title{
Numerical simulations of negatively buoyant jets in an immiscible fluid using the Particle Finite Element Method
}

\author{
M. Mier-Torrecilla ${ }^{1, *, \dagger}$, A. Geyer ${ }^{1}$, J. C. Phillips ${ }^{2}$, S.R. Idelsohn ${ }^{1, \dagger}$ and E. Oñate $^{1}$ \\ ${ }^{1}$ CIMNE International Center for Numerical Methods in Engineering, 08034 Barcelona, Spain \\ ${ }^{2}$ Department of Earth Sciences, University of Bristol, Bristol BS8 1RJ, UK
}

\begin{abstract}
SUMMARY
Negatively buoyant jets consist in a dense fluid injected vertically upward into a lighter ambient fluid. The numerical simulation of this kind of buoyancy-driven flows is challenging as it involves multiple fluids with different physical properties. In the case of immiscible fluids, it requires, in addition, to track the motion of the interface between fluids and accurately represent the discontinuities of the flow variables.

In this paper, we investigate numerically the injection of a negatively buoyant jet into a homogenous immiscible ambient fluid using the Particle Finite Element Method and compare the two-dimensional numerical results with experiments on the injection of a jet of dyed water through a nozzle in the base of a cylindrical tank containing rapeseed oil. In both simulations and experiments, the fountain inlet flow velocity and nozzle diameter have been varied to cover a wide range of Froude $F r$ and Reynolds $R e$ numbers $(0.1<F r<30,8<R e<1350)$, reproducing both weak and strong laminar fountains.

The flow behaviors observed for the different numerical simulations fit in the regime map based on the $R e$ and $F r$ values of the experiments, and the maximum fountain height is in good agreement with the experimental observations, suggesting that particle finite element method is a useful tool for the study of immiscible two-fluid systems. Copyright () 2011 John Wiley \& Sons, Ltd.
\end{abstract}

Received 22 December 2010; Revised 2 May 2011; Accepted 12 May 2011

KEY WORDS: negatively buoyant jets; fountain; particle finite element method; interfacial tension; immiscible fluids

\section{INTRODUCTION}

When a dense fluid is injected vertically upwards into a lighter ambient fluid, its momentum is continually being decreased by buoyancy forces until the vertical velocity becomes zero at some finite distance from the source. As the jet reaches its maximum penetration length $h_{\max }$, it reverses its direction and flows back in an annular geometry around the upflow (Figure 1). Such jets are called negatively buoyant jets or fountains, and the density difference between the ambient and the injected fluids may be due to a variation in either chemical composition or temperature.

Negatively buoyant jets or fountains are common both in engineering and natural science. An everyday example is the ventilation of large open structures such as aircraft hangars, which are heated using ceiling-mounted fans to drive hot air towards the floor. In nature, geophysical buoyant jets resulting from temperature (or salinity) differences can occur in magma chambers and in the ocean (e.g., $[1,2])$.

During the last 50 years, the behavior of negatively buoyant jets or fountains has been widely explored theoretically and experimentally (e.g., [3-16]). Since the pioneering work of Morton [3],

\footnotetext{
*Correspondence to: M. Mier-Torrecilla, CIMNE International Center for Numerical Methods in Engineering, 08034 Barcelona, Spain.

${ }^{\dagger}$ E-mail: mdemier@cimne.upc.edu

*ICREA research professor at CIMNE. 


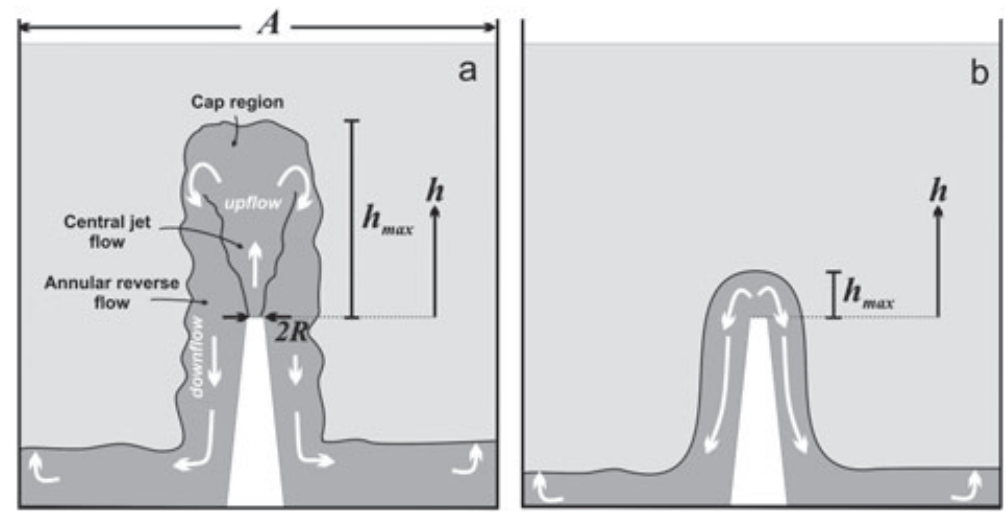

Figure 1. Sketch of a strong (a) and a weak (b) fountain. Description of the different parameters is found in Table II and the text.

significant progress has been made in understanding the dynamics of negatively buoyant jets arriving at a general description of their flow behavior, summarized in the next section.

Currently, only limited numerical simulations of the dynamics of negatively buoyant jets have been presented [17-24] because they still pose a major research challenge from both theoretical and computational point of view. These studies performed direct numerical simulations of thermal axisymmetric and plane fountains using the finite volume method; being the cause of the density gradient between both fluids is the difference in temperature.

When modeling immiscible fluids, the dynamics of the interface between fluids play a dominant role. The success of the simulation of such flows depends on the ability of the numerical method to model accurately the interface and the phenomena taking place on it, such as the surface tension. Therefore, in addition to the well-known numerical difficulties in the simulation of single-fluid flows (namely, the coupling of pressure and velocity through the incompressibility constraint, the need of the discretization spaces to satisfy the inf-sup condition, and the nonlinearity of the governing equations), the computation of immiscible multi-fluid flows faces three major challenges:

1. Accurate description of the interface position.

The location of the interface separating the fluids is in general unknown and coupled to the local flow field, which transports the interface. The interface needs to be tracked accurately without introducing excessive numerical smoothing, and it is essential that the interface is able to fold, break, and merge.

2. Modeling the jumps in the fluid properties and flow variables across the interface. Jumps of the fluid density and viscosity across the interface, paralleled by discontinuities in pressure and stresses, need to be properly taken into account to satisfy the momentum balance at the vicinity of the interface.

3. Modeling the surface tension.

Because surface tension plays a very important role in the immiscible interface dynamics, this force needs to be accurately evaluated and incorporated into the model.

In their recent work, Mier-Torrecilla et al. [25] developed a numerical scheme for the simulation of multi-fluid flows with the particle finite element method (PFEM) able to deal with immiscible fluids. Here, we go a step further and apply the scheme to investigate the dynamics of negatively buoyant jets in a homogenous immiscible ambient fluid. For this purpose, we use the PFEM to solve the isothermal, incompressible Navier-Stokes equations in two dimensions. The simulations include the effect of surface tension and the sharp jumps in the density and viscosity of the fluids at the interface. The simulated process corresponds to the continuous injection of a liquid (water) into a cylindrical container filled with a more viscous immiscible liquid (rapeseed oil), through a conical nozzle located at the base of the oil container. In the different numerical runs, we have varied the injection velocity and the nozzle radius to reproduce a wide range of Reynolds $R e$ and Froude $\mathrm{Fr}$ numbers. In contrast to previous published results (Table I), numerical simulations presented in this 
paper cover a larger Froude number interval, $0.1<F r<30$, being able to reproduce both weak and strong fountains in a laminar regime $(8<R e<1350)$. We compare the numerical predictions with experimental observations outcomes from a more detailed experimental study by Geyer et al. [26].

\section{NEGATIVELY BUOYANT JETS}

The flow behavior of negatively buoyant jets may be summarized as follows. The jet penetrates initially to a maximum height $h_{\max }$ in the tank, which depends on the initial upward momentum and the opposing downward negative buoyancy force. Then, the jet collapses decreasing its penetration height and reaching a steady state where the penetration depth remains constant and slightly smaller than $h_{\max }$. In negatively buoyant jets, three flow regimes can be distinguished (Figure 1(a)) [27]: the central jet flow, the annular reverse flow, and the 'cap' region where the large-scale reversal of fluid takes place.

How the flow behaves in detail depends on the following factors [4, 16, 27]: jet parameters, environmental parameters, and geometrical factors. The first group of parameters includes the initial jet velocity distribution and turbulence level (whether the jet is laminar or turbulent), as well as the mass, momentum, and buoyancy fluxes. The fountain can be described as strong or weak depending on the ratio of buoyancy and momentum flux, or if the fountain is laminar or turbulent. For strong fountains (the discharge momentum is relatively larger than the negative buoyancy of the flow), the fountain top, plunging plume, and intrusion flow are distinct features (Figure 1(a)). Kinetic energy is converted into potential energy until $h_{\max }$ is reached and then the fluid begins to accumulate at the top of the fountain. As the mass of accumulated fluid increases, eventually the downward buoyancy force exceeds the inertia of the jet and the collapse occurs. When the falling fluid collapses back to the level of the nozzle, it dislodges from the jet and a new cycle begins. If the source momentum is further increased, this oscillatory behavior persists at increasing amplitudes until a second threshold limit is reached above, which the fountain no longer exhibits high-amplitude pulsations [7]. For weak fountains (discharge inertia of the fountains is equal or less than the negative buoyancy force), the fluid exiting the fountain remains attached to the nozzle because of capillary and gravity forces, that is, the upward and downward flows cannot be visually distinguished. Instead, the streamlines curve and spread from the source and fountain top (Figure 1(b)). The second group of variables, environmental parameters, includes parameters describing the ambient fluid (e.g., turbulence level, any net flow, and density stratification), and the geometrical factors include the jet shape, its orientation, and proximity to solid boundaries or to the free surface.

The most common dimensionless numbers applied to the study of negatively buoyant jets are summarized in Table II. The Reynolds number $(R e)$ characterizes the ratio between inertia and viscous effects in the flow at the nozzle. The Froude number $(F r)$ compares kinetic energy with gravitational energy, and this ratio has also been expressed by some authors [12-14] as the Richardson number $R i$, being $R i=\mathrm{Fr}^{-2}$. Furthermore, interfacial tension effects and characteristic frequency for the flow structure are nondimensionalized in the Weber $(W e)$ and Strouhal $(S t r)$ numbers, respectively. Phase-mingling onset conditions and characteristic diameters are nondimensionalized in the Bond number $(B o)$.

Table I. Summary of previous numerical models for negatively buoyant jets.

\begin{tabular}{lllc}
\hline Reference & \multicolumn{1}{c}{ Jet flow } & Range of $F r$ & Range of $R e$ \\
\hline$[17]$ & laminar/very weak & $0.0025 \leqslant F r \leqslant 0.2$ & $5<\operatorname{Re}<800$ \\
{$[18]$} & laminar/weak & $0.2 \leqslant F r \leqslant 1.0$ & $\operatorname{Re}=200$ \\
{$[19,20]$} & laminar/weak & $0.2 \leqslant F r \leqslant 1.0$ & $5 \leqslant \operatorname{Re} \leqslant 200$ \\
{$[21]$} & laminar/weak & $F r=1$ & $5 \leqslant R e \leqslant 800$ \\
{$[22]$} & laminar/weak-forced & $1 \leqslant F r \leqslant 8$ & $200 \leqslant R e \leqslant 800$ \\
{$[23]$} & laminar/very weak-forced & $0.25 \leqslant F r \leqslant 10.0$ & $\operatorname{Re}=100$ \\
{$[24]$} & turbulent/very weak-forced & $0.45<F r<2.2$ & $\operatorname{Re}=3350$ \\
\hline
\end{tabular}


Table II. List of variables, parameter values and their SI units, and dimensionless groups.

\begin{tabular}{|c|c|c|c|}
\hline$A$ & Container diameter & 0.1 & $\mathrm{~m}$ \\
\hline$d$ & Nozzle diameter (in numerical models) & $2.62 \times 10^{-3} / 3.5 \times 10^{-3}$ & $\mathrm{~m}$ \\
\hline$R$ & Nozzle radius & $d / 2$ & $\mathrm{~m}$ \\
\hline$g$ & Gravity & 9.81 & $\mathrm{~m} \mathrm{~s}^{-2}$ \\
\hline$g^{\prime}$ & Reduced gravity & $g^{\prime}=g \frac{\rho_{j}-\rho_{a}}{\rho_{i}}$ & $\mathrm{~m} \mathrm{~s}^{-2}$ \\
\hline$h_{\max }$ & Maximum penetration depth & ro & $\mathrm{m}$ \\
\hline$n$ & Normal direction & & - \\
\hline$Q$ & Volumetric flow rate & & $\mathrm{m}^{3} \mathrm{~s}^{-1}$ \\
\hline$u_{j}$ & Vertical jet velocity & & $\mathrm{m} \mathrm{s}^{-1}$ \\
\hline $\bar{u}$ & Average vertical velocity & & $\mathrm{m} \mathrm{s}^{-1}$ \\
\hline$u^{*}$ & Characteristic jet velocity & & $\mathrm{m} \mathrm{s}^{-1}$ \\
\hline$\rho_{a}$ & Density of the ambient fluid (rapeseed oil) & 900 & $\mathrm{~kg} \mathrm{~m}^{-3}$ \\
\hline$\rho_{j}$ & Density of the jet fluid (water) & 1000 & $\mathrm{~kg} \mathrm{~m}^{-3}$ \\
\hline$\gamma$ & Interfacial tension coefficient (water-rapeseed oil) & 0.02 & $\mathrm{~N} \mathrm{~m}^{-1}$ \\
\hline$\mu_{a}$ & Dynamic viscosity of the ambient fluid (rapeseed oil) & $200 \times 10^{-3}$ & Pa s \\
\hline$\mu_{j}$ & Dynamic viscosity of the injected fluid (water) & $10^{-3}$ & Pa s \\
\hline$f$ & Characteristic frequency of the flow structure & & $\mathrm{s}^{-1}$ \\
\hline Bo & Bond number : gravity versus surface tension & $\mathrm{Bo}=\frac{R^{2} g\left(\rho_{j}-\rho_{a}\right)}{2}$ & \\
\hline$F r$ & Froude number: inertia versus buoyancy & $F r={\frac{u_{j}}{\sqrt{R g^{\prime}}}}^{\gamma}$ & \\
\hline$H_{\max }$ & Dimensionless $h_{\max }$ & $H_{\max }=\frac{h_{\max }}{R}$ & \\
\hline $\operatorname{Re}$ & Reynolds number: inertia versus viscosity & $R e=\frac{\rho_{j} u_{j} R}{\mu_{j}}$ & \\
\hline$R i$ & Richardson number: buoyancy versus inertia & $R i=\frac{R g^{\prime}}{u_{j}^{2}}=F r^{-2}$ & \\
\hline Str & Strouhal number: oscillations frequency & $S t r=\frac{f R}{u_{j}}$ & \\
\hline$W e$ & Weber number: inertia versus surface tension & $W e=\underline{\rho_{a} u_{j}^{2} R}$ & \\
\hline & & $\gamma$ & \\
\hline
\end{tabular}

\section{NUMERICAL METHODOLOGY: THE PARTICLE FINITE ELEMENT METHOD}

The PFEM [28-30] is a numerical technique for modeling and analysis of complex multidisciplinary problems in fluid and solid mechanics, including thermal effects and interfacial or free-surface flows, among others. In the last years, the PFEM has been successfully applied to naval and coastal engineering [31-33], fluid-structure interaction [34-36], melting of polymers in fire [37], and multi-fluid flows [38-40].

Particle finite element method is a particle method in the sense that the domain is defined by a collection of particles that move in a Lagrangian manner according to the calculated velocity field, transporting their momentum and physical properties (e.g., density and viscosity). The interacting forces between particles are evaluated with the help of a mesh. Mesh nodes coincide with the particles so that when the particles move, so does the mesh. On this moving mesh, the governing equations are discretized using the standard FEM. The possible large distortion of the mesh is avoided through remeshing of the computational domain. A robust and efficient Delaunay triangulation algorithm allows frequent remeshing. This gives the method excellent capabilities for modeling large displacement and large deformation problems.

In the case of multi-fluid flows, the dynamics of the interface between fluids play a dominant role. The success of the simulation of such flows depends on the ability of the numerical method to model accurately the interface and the phenomena taking place on it, such as the surface tension. In a previous paper [25], we described in detail the governing equations and their numerical solution 
via PFEM and demonstrated that the method accurately simulates multi-fluid flows, in particular, the rising bubble problem. Here, we apply it to the more complex problem of negatively buoyant jets, where frequent breakup and coalescence of fluid regions and a much larger interface deformation take place. The main aspects of the algorithm are summarized in the following text.

\subsection{Solution scheme}

A typical PFEM solution scheme requires five different steps for each timestep (Figure 2): step 1, definition of the set of nodes in the fluid and solid domains (Figure 2(b)); step 2, identification of the external boundary and the internal interfaces because some boundaries/interfaces may be severely distorted during the solution process (Figure 2(c)); step 3, discretization of the domain with a finite element mesh generated by Delaunay triangulation (Figure 2(d)); step 4, solution of the Lagrangian-governing equations of motion for the fluid domain together with the boundary and interface conditions (Figure 2(e)); and step 5, moving the mesh nodes to a new position based on the time increment and the velocity field computed in step 4 (Figure 2(f)). After step 5, a new timestep starts.

As in standard FEM, the accuracy of PFEM solutions depends on the discretization size. The mesh is refined close to the interface to improve the accuracy, and an adaptive timestep algorithm ensures selection of the largest timestep that avoids element inversion. Linear shape functions are used for all unknowns to increase computational efficiency. Pressure is stabilized with a pressure gradient projection method, and it is decoupled from the velocity in the momentum equation through a second-order fractional-step scheme. The Lagrangian formulation eliminates the convective terms in the governing equations (and thus circumvents the numerical difficulties associated with convection) and naturally tracks the motion of interfaces. Refer to [25] for further details about the algorithm and convergence analysis.

Regarding multi-fluid flow simulation, the key points of the algorithm are the following:

1. The interface is described by mesh nodes and element edges (Figure 2(d)), and thus it is a well-defined curve, with the information regarding its location and curvature readily available.

2. The interface nodes carry the jump of properties (density and viscosity), maintaining the interface sharp along time so that it is clear which property value is valid at each point of the domain.

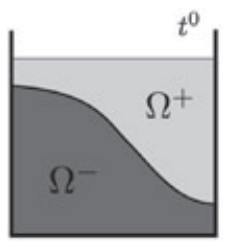

(a)

)

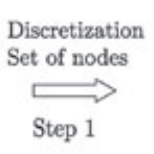

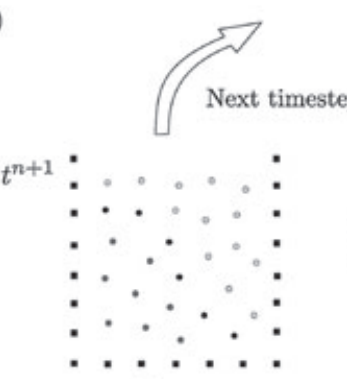

(f)

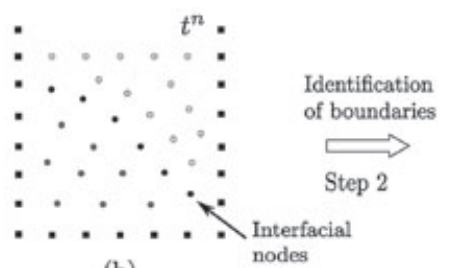

(b)

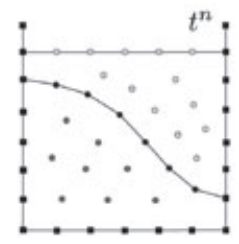

(c)

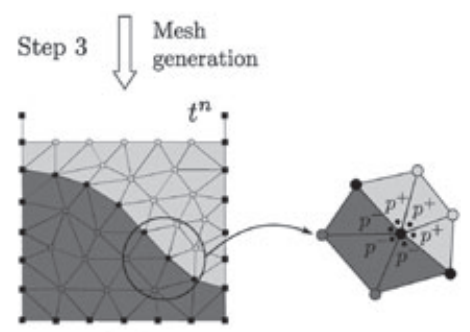

(d)

Figure 2. Particle finite element method solution steps illustrated in a simple dam-break example. As the gate of the dam is removed, the water begins to flow. (a) Continuous problem; (b) Step 1, discretization in cloud of nodes at time $t^{n}$; (c) Step 2, boundary and interface recognition; (d) Step 3, mesh generation and detail of the duplicated pressure degrees of freedom at the interface; (e) Step 4, resolution of the discrete governing equations; (f) Step 5, nodes moved to new position for time $t^{n+1}$. 
3. Kinks ( $\mathcal{C}^{0}$ discontinuities) in the solution are automatically represented when the interface is aligned with the mesh. Only jumps $\left(\mathcal{C}^{-1}\right.$ discontinuities) need some attention in the PFEM. In particular, the pressure field has been made double valued at the interface, that is, pressure DOFs have been duplicated $\left(p^{+}, p^{-}\right)$in the interface nodes, to represent the pressure discontinuity caused by the jump in viscosity and/or surface tension (Figure 2(d)).

4. Surface tension force is applied directly on the interface nodes, avoiding the need of regularization techniques.

The mesh is refined close to the interface to improve the curvature calculation for the surface tension force. The medial axis technique [40] is used to compute the distance to the interface and prescribe an element size $\Delta x$ proportional to this distance. Mesh adaptivity allows to improve the accuracy (finer interface representation) and the efficiency (refinement only where required) of the method. Thus, mesh refinement is dynamical and adapts to the current interface position.

Interface nodes are identified as those belonging simultaneously to elements of different fluids. Interfacial boundary conditions (i.e., continuity of all velocity components and normal stresses balanced with the surface tension force) are applied on these nodes, and because of the duplicated pressure DOFs, the pressure vector in the matrix system has double number of entries for these nodes.

For the surface tension force at the interface, $\gamma \kappa \boldsymbol{n}$, the curvature $\kappa$ and the unitary normal direction $\boldsymbol{n}$ are calculated from the information of the interface location using the osculating circle of a curve [25], as illustrated in Figure 3. In this way, $\boldsymbol{n}=\frac{\boldsymbol{R}}{|\boldsymbol{R}|}$ and $\kappa \boldsymbol{n}=\frac{\boldsymbol{R}}{|\boldsymbol{R}|^{2}}$.

\section{NUMERICAL SIMULATIONS OF NEGATIVELY BUOYANT JETS}

Numerical simulation of negatively buoyant jets involves fluids with considerably different physical properties and complex interfacial phenomena such as surface tension and changes in topology (e.g., jet breakup in drops or coalescence of bubbles). In this paper, we use the PFEM to simulate the injection of dyed freshwater through a re-entrant nozzle in the base of a cylindrical tank containing rapeseed oil. The physical properties of the fluids are listed in Table II. The computational domain consists of a container of finite height and width with no-slip side and bottom walls and open top. For the numerical study, the injection of the dense fluid (water) is simulated as arising because of the motion of a solid piston, leading to uniform source velocity profiles. The element size of the unstructured triangular mesh is $\Delta x=0.002 \mathrm{~m}$ (50 elements along the container width) and has been refined to $\Delta x / 4$ at the interface (Figure 4).

Previous numerical studies (Table I) have performed direct numerical simulation of thermal axisymmetric and plane fountains up to $F r=10$ using the finite volume method. In these studies, the density gradient between fluids is due to a difference in temperature (therefore, the density jump between fluids is smoothed by the thermal diffusion), and viscosity is considered equal in both fluids. Furthermore, they do not take the role of interfacial tension into account, whereas in the

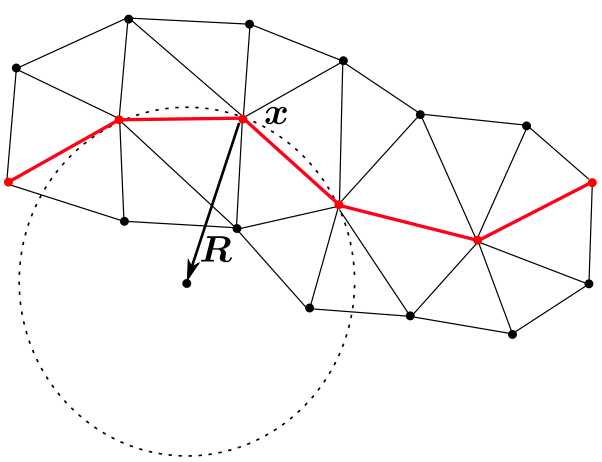

Figure 3. Calculation of the osculating circle at node $\boldsymbol{x}$. 


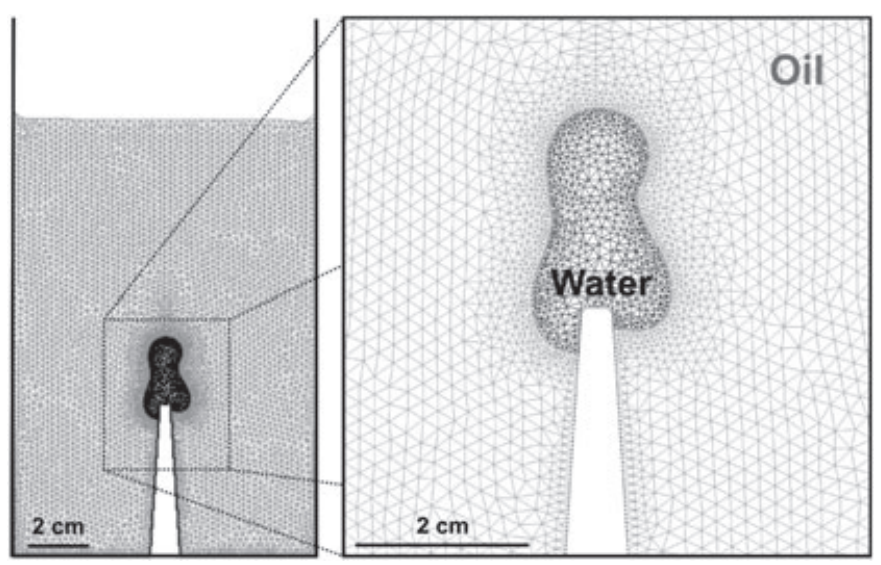

Figure 4. Detail of the unstructured mesh refined close to the interface.

simulations presented here, the participating fluids are immiscible (with realistic physical properties and non-negligible surface tension) and therefore, the interface needs to be accurately tracked. Furthermore, the simulations cover a larger Froude number interval $(0.1<F r<30)$ and reproduce both weak and strong fountains in a laminar regime $(8<R e<1350)$.

We have performed 12 two-dimensional numerical simulations, which are compared with more than 70 experimental results of a parallel study by Geyer et al. [26]. In the different numerical runs, we have varied the injection velocity and the nozzle radius to reproduce the wide range of Reynolds and Froude numbers covered by the experiments. The PFEM simulations, together with the corresponding values for the dimensionless maximum height $H_{\max }, F r$, and $R e$, are listed in Table III. Figure 5 shows the values of $F r$ and $R e$ for both the numerical and the experimental runs and includes also the ranges covered by previous numerical studies.

\subsection{Evolution of the fountain flow}

Particle finite element method simulates the evolution of the flow at each timestep, which allows us to describe the process for any $(F r, R e)$ pair. Figures 6 and 7 illustrate the results obtained for increasing $F r$ values. For low $F r$ and $R e$ numbers (simulation $\mathrm{C}, \mathrm{Fr}=0.6, \operatorname{Re}=43.75$ ) (Figure 6(a)), we observe a weak fountain with almost indistinguishable upward and downward flow. The fluid exiting the nozzle remains attached to the nozzle because of capillary and gravity forces. However, as the jet velocity is increased, that is, increasing $F r$ and $R e$ (simulation $\mathrm{F}$, $F r=5.57, R e=262.5$ ) (Figure 6(b)), the jet penetrates upward into the ambient liquid reaching $h_{\text {max }}$ shortly after the start. Because of the fact that the simulations are two dimensional, some oil

Table III. Settings of the numerical simulations. Values for the different variables and related dimensionless numbers are also included.

\begin{tabular}{lccccc}
\hline Simulation & $R(\mathrm{~m})$ & $u\left(\mathrm{~m} \mathrm{~s}^{-1}\right)$ & $H_{\max }$ & $F r$ & $R e$ \\
\hline A & $1.75 \times 10^{-3}$ & 0.005 & 2.25 & 0.12 & 8.75 \\
$\mathrm{~B}$ & $1.75 \times 10^{-3}$ & 0.01 & 3.24 & 0.24 & 17.50 \\
$\mathrm{C}$ & $1.75 \times 10^{-3}$ & 0.025 & 2.74 & 0.60 & 43.75 \\
$\mathrm{D}$ & $1.75 \times 10^{-3}$ & 0.05 & 3.7 & 1.21 & 87.50 \\
$\mathrm{E}$ & $1.75 \times 10^{-3}$ & 0.1 & 6.04 & 2.41 & 175.00 \\
$\mathrm{~F}$ & $1.31 \times 10^{-3}$ & 0.2 & 18.58 & 5.57 & 262.50 \\
$\mathrm{G}$ & $1.31 \times 10^{-3}$ & 0.3 & 23.9 & 8.36 & 393.75 \\
$\mathrm{H}$ & $1.31 \times 10^{-3}$ & 0.4 & 43.28 & 11.15 & 525.00 \\
$\mathrm{I}$ & $1.31 \times 10^{-3}$ & 0.5 & 66.82 & 13.93 & 656.25 \\
$\mathrm{~J}$ & $1.31 \times 10^{-3}$ & 0.6 & 76.58 & 16.72 & 787.50 \\
$\mathrm{~K}$ & $1.31 \times 10^{-3}$ & 0.8 & - & 22.29 & 1050.0 \\
$\mathrm{~L}$ & $1.31 \times 10^{-3}$ & 1.0 & - & 27.87 & 1312.50 \\
\hline
\end{tabular}




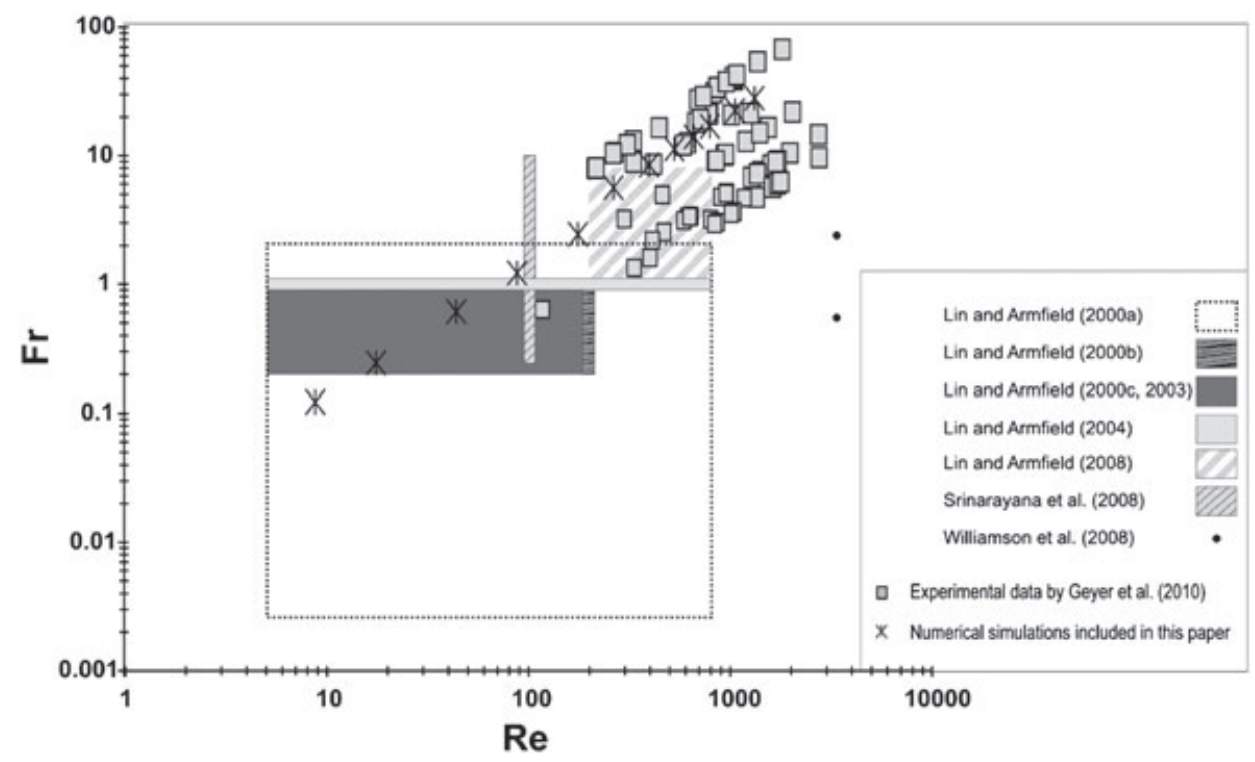

Figure 5. Fr-Re regime diagram showing the parameter ranges investigated by previous numerical studies. The $(F r, R e)$ pair values for both the numerical and the experimental studies presented in this paper are shown as symbols.

regions get enclosed by water. These oil bubbles are lighter and therefore move upward until they merge with the bulk oil. In Figure 7, we have included results for two simulations with high-inlet velocity. In case of simulation I $\left(u_{j}=0.5 \mathrm{~m} \mathrm{~s}^{-1}, F r=13.93, R e=656.25\right)$ (Figure 7(a)), a cap forms when the jet reaches $h_{\max }$, spreading radially as it is supplied with negatively buoyant jet fluid. In this simulation, we observe that the fountain flow emanating from the nozzle may be divided into a 'smooth' part and a 'wavy' one (Figure 7(a)). The instability of the flow that leads to this transformation is due to the Kelvin-Helmholtz instability that occurs at the shear layer between the jet and the ambient fluid. As the injection velocity is further increased (simulation $\mathrm{L}, u_{j}=1.0 \mathrm{~m} \mathrm{~s}^{-1}$ ) (Figure 7(b)), the injected fluid breaks into droplets. In both simulations of Figure 7, it can be seen that some of the drops remain attached to the central cap region. This phenomenon is not a numerical artefact. It is also observable in the experimental results (see Figure 10 in Section 4.3).

\subsection{Fountain penetration height and velocity profiles}

The time evolution of the fountain height $h$ for simulations A-G is presented in Figure 8. For the simulations with lower $F r$ numbers (simulations A-E), it can be seen that after reaching $h_{\max }$, the jet height decreases and remains statistically stable at a lower height. Oscillations are mainly due to capillary waves that propagate along the oil-water interface. As the injection velocity is increased (simulations $\mathrm{F}$ and $\mathrm{G}$ ), this behavior changes, and after reaching $h_{\max }$, the flow does not stabilize. Furthermore, in simulation F, the flow oscillates in both a high-frequency and a low-frequency mode. In the case of simulations $\mathrm{H}-\mathrm{L}$, it is not possible to precisely define $h$ because of the breakup of the jet into drops.

Figure 9 shows the vertical velocity field for simulations F, I, and L. Upflow and downflow can be clearly identified in simulation $\mathrm{F}$. The velocity profile also shows that the vertical velocity is nearly constant outside the jet. In strong fountains (simulations I and L), the ambient fluid is transported upward by the shear against the jet, and it can be observed that the flow accelerates in the narrower regions of the jet. The velocity profile is almost uniform close to the source because of the fact that the injection has been implemented as a piston.

\subsection{Comparison of numerical results with experimental data}

To validate the PFEM simulations, the numerical results obtained have been compared with a series of previous laboratory experiments, where a dense liquid was injected through a nozzle into the 
a)

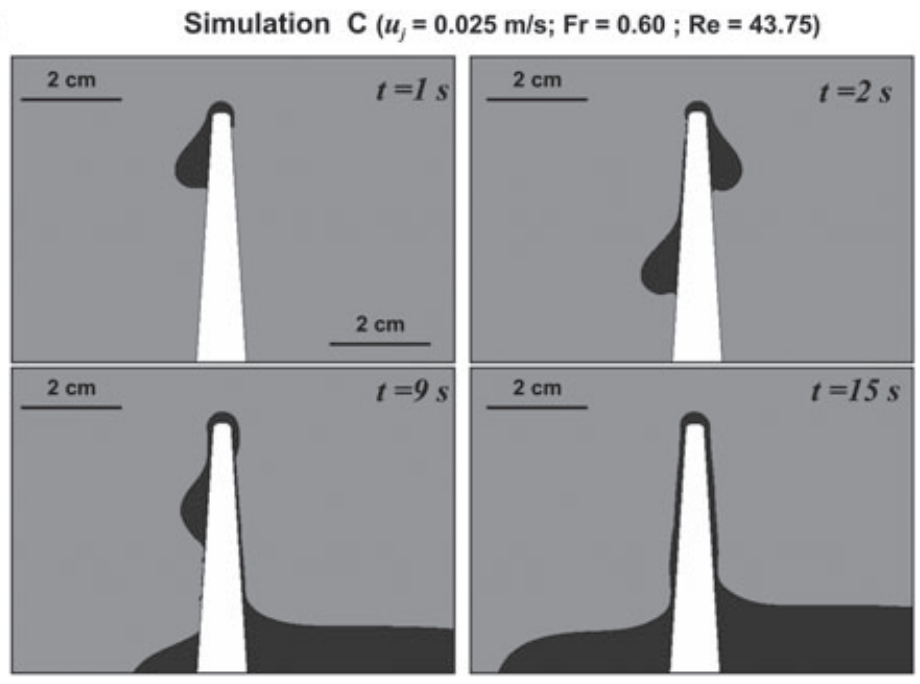

b)

Simulation $\mathrm{F}\left(u_{j}=0.2 \mathrm{~m} / \mathrm{s} ; \mathrm{Fr}=5.57 ; \mathrm{Re}=262.5\right)$
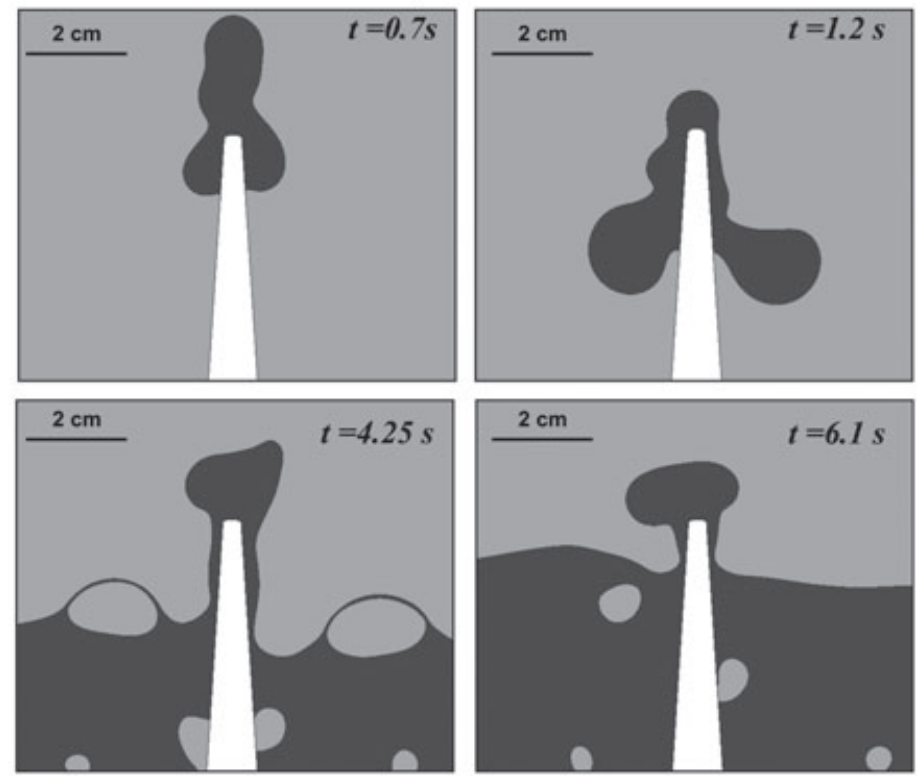

Figure 6. Flow evolution for simulations $\mathrm{C}$ and $\mathrm{F}$ (very weak and weak fountain, respectively). The animation corresponding to these two simulations may be found in the online version of this paper.

base of a cylindrical tank containing a less dense, immiscible liquid. Full details concerning the experimental apparatus, method, and the main results obtained are available in Geyer et al. [26].

In short, experimental results show that, for a given fountain geometry, three different flow regimes are recognizable as the inlet volumetric flow rate is increased (Figure 10). Flow regime type I is observed at low source volumetric flow rates and is characterized by an approximately constant fountain height, within the range of experimental error of the observation. As the source volumetric flow rate is increased, the fountain height is not constant but is characterized by continuously varying with time $t$ between a maximum $h_{\max }$ and a minimum height $h_{\min }$; this is type II (pulsating) behavior. As the source volumetric flux and velocity is further increased, type III behavior is observed. The jet initially penetrates upward into the ambient fluid, and when it reaches $h_{\max }$, a 'cap' forms of accumulated jet fluid at the top of the jet. The size of this cap increases because of the continuous fluid supply from the fountain, but its vertical position remains constant at $h_{\max }$. 
a)

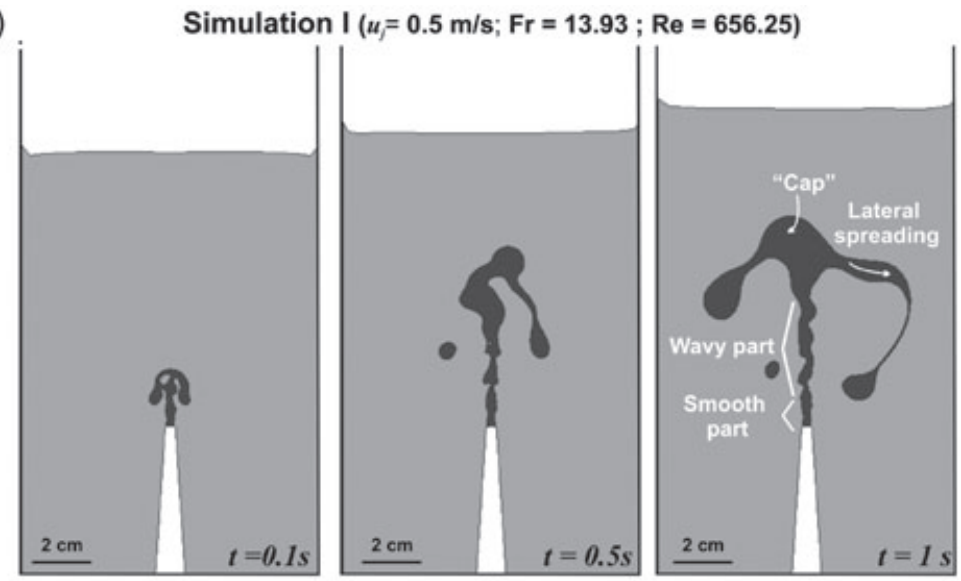

b)

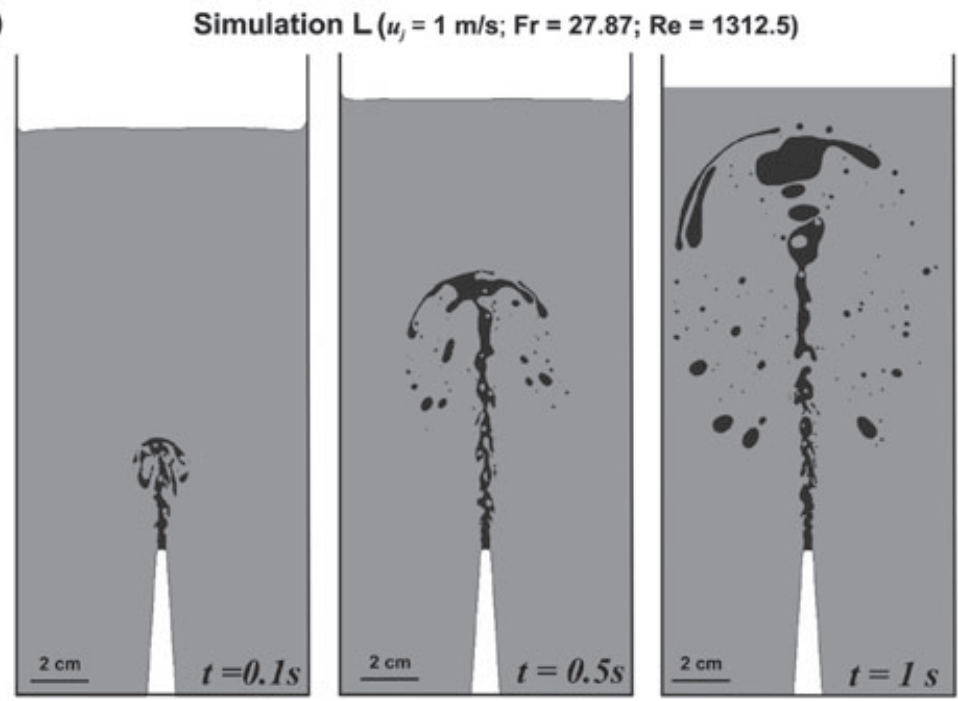

Figure 7. Flow evolution for simulations I and L (both strong fountains). The animation corresponding to these two simulations may be found in the online version of this paper.

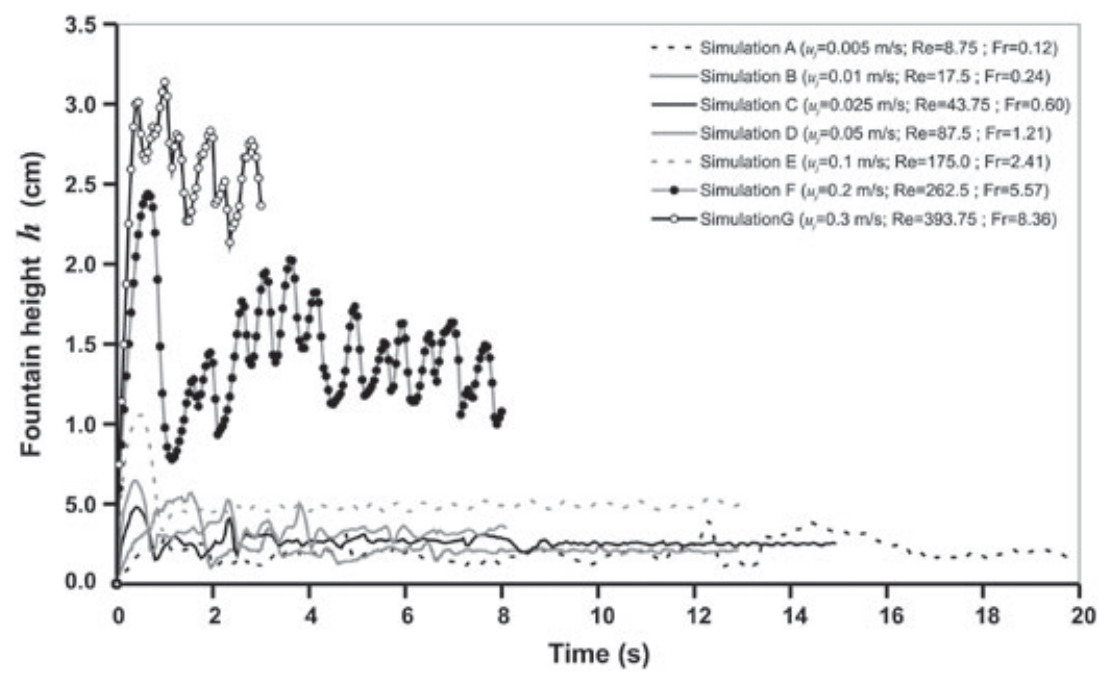

Figure 8. Fountain height versus time for various numerical simulations. 

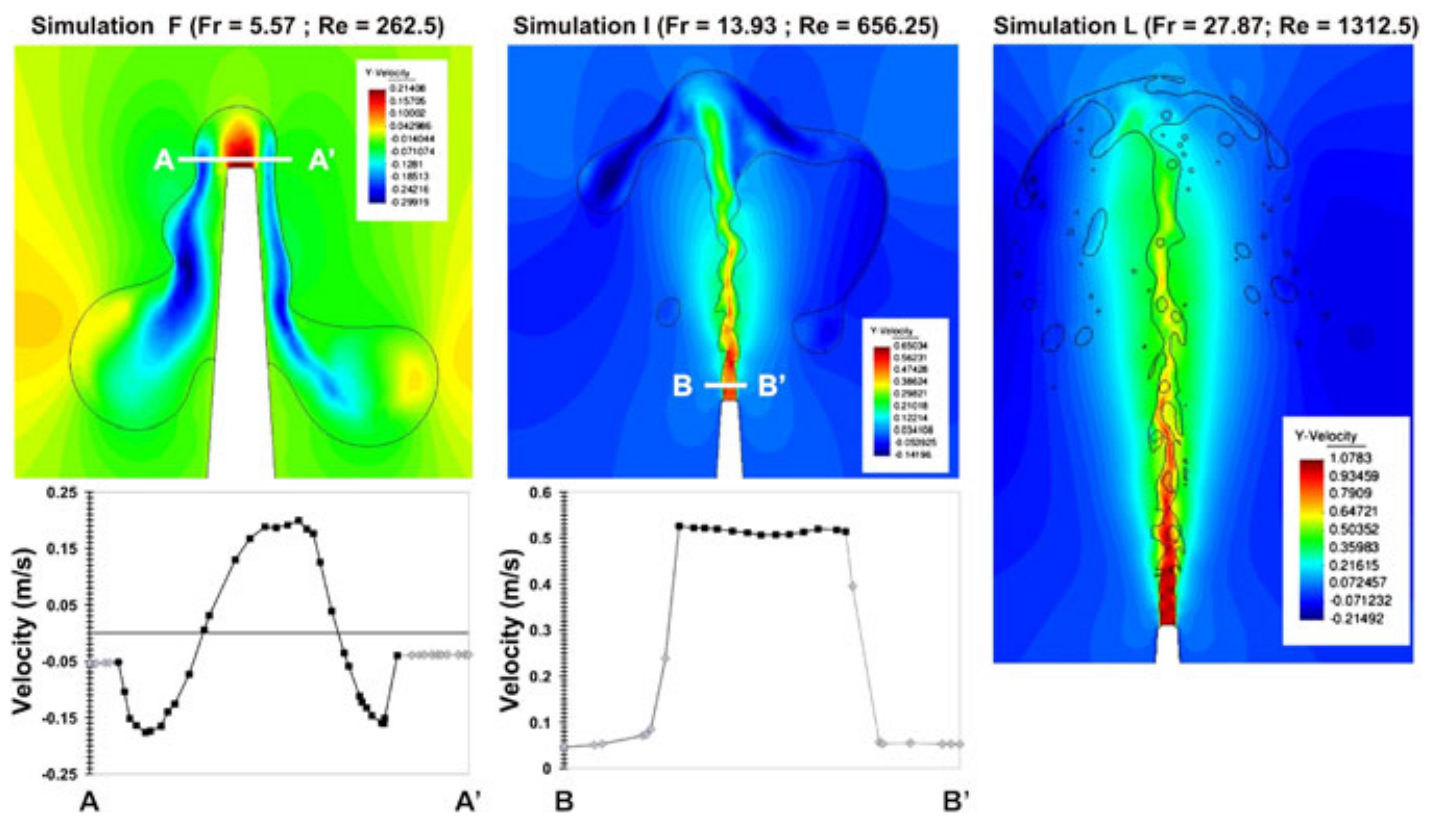

Figure 9. Vertical velocity field for simulation $\mathrm{F}$ (at time $t=1.2 \mathrm{~s}), \mathrm{I}(t=1.0 \mathrm{~s})$, and $\mathrm{L}(t=0.5 \mathrm{~s})$ and profile along the cross sections $\mathrm{AA}^{\prime}$ and $\mathrm{BB}^{\prime}$ ( $2 \mathrm{~mm}$ above the nozzle).
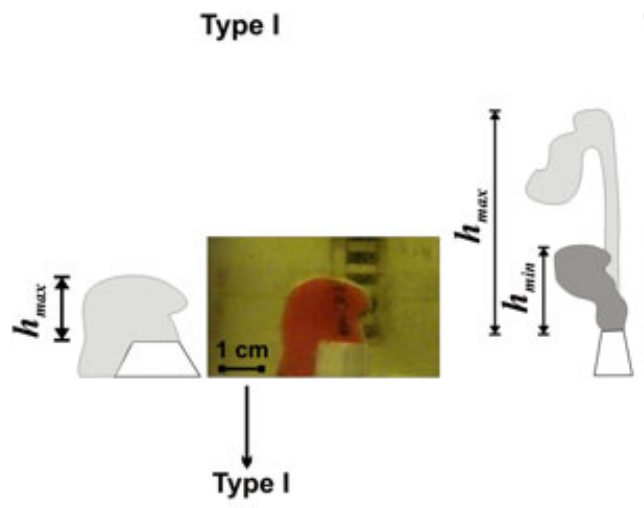

Type II

Type III
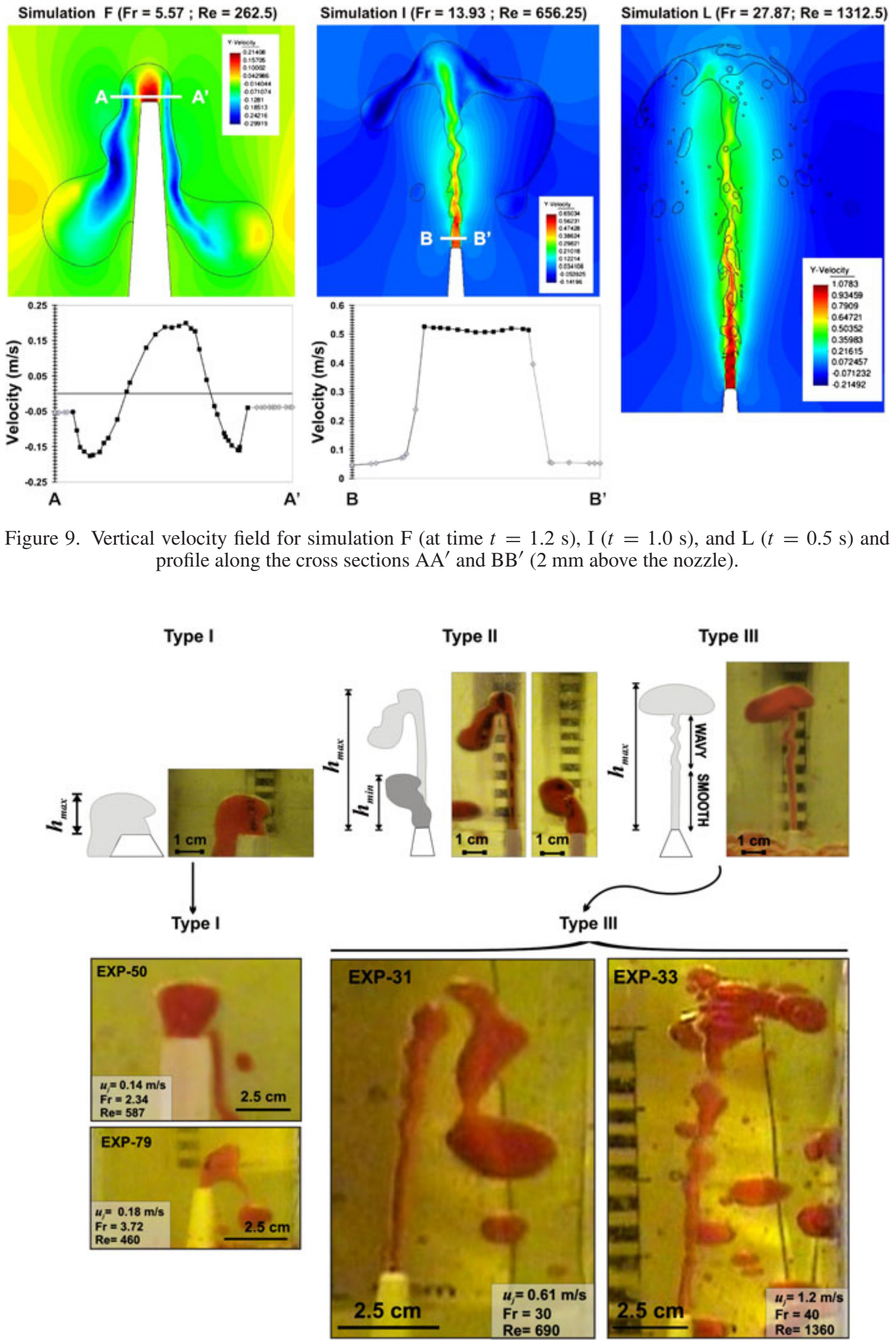

$$
\text { , }
$$


Once the cap exceeds a critical size, it breaks up, and water droplets fall back to the base of the tank. In this regime, the fountain is characterized by a smooth and a wavy part (Figure 10).

Based on the $R e$ and $F r$ values for the experimental simulations, Geyer et al. [26] were able to provide a regime map to define how these dimensionless numbers may control the occurrence of each of the observed flow types (Figure 11). Fr may play a stronger role in comparison with $R e$ in determining the maximum penetration height. By contrast, the effect of $R e$ may be stronger than $F r$ s in providing a prediction of the flow behavior for a specific nozzle diameter and injection velocity. The transition between types I and II appears to be uniquely controlled by the $F r$ number, whereas it is possible to pass from type I or II to type III by increasing $R e$ alone.

If we compare our numerical simulations with the experimental results, we find a quite acceptable agreement within the range of the experimental error of the observation. In Figure 11, we have plotted also the $(F r, R e)$ pairs of the performed numerical simulations (A to L) (Table I). From the regime map, we should expect that simulations A to E show type I behavior, simulations $\mathrm{F}$ to $\mathrm{H}$ have type II behavior, and simulations I to L type III. Comparing Figures 6, 7, and 10, we can see that our expectations are accomplished. In particular, the fountain of simulation $\mathrm{C}$ matches type I behavior (compare Figures 6(a) and 10), simulation F shows the same type of fluctuations in height as those observed for type II fountains (compare Figures 6(b), 8, and 10), and simulations I and L behave similarly to type III fountains (compare Figures 7 and 10).

However, the correspondence between the numerical and the physical modeling is not limited to qualitative observations. In Figure 12, we have plotted the dimensionless maximum fountain height, $H_{\max }$, against $F r$ and $R e$ for all types II and III numerical simulations and the experiments. As for the $(F r, R e)$ plot, we observe excellent correlation between the numerical simulations and the experimental observations in terms of the different flow types.

The comparison between the numerical and experimental data offered in this section has demonstrated that PFEM is suitable for studying this kind of flows. Although the numerical simulations are run in $2 \mathrm{D}$ and the presented experiments are $3 \mathrm{D}$, the main variable chosen for comparison $\left(h_{\max }\right)$ is few affected by three-dimensional effects as previous studies $[4,17,19,20]$ suggest that the maximum penetration height is mainly related to the momentum and buoyancy fluxes at the source (expressed in terms of $\mathrm{Fr}$ and $\mathrm{Re}$ ) and not so much to the surface tension. In other kind of analyses involving the amount of drops formed, their size, or the time of breakup, three-dimensional phenomena would be determinant in the results. We are aware that two-dimensional computations

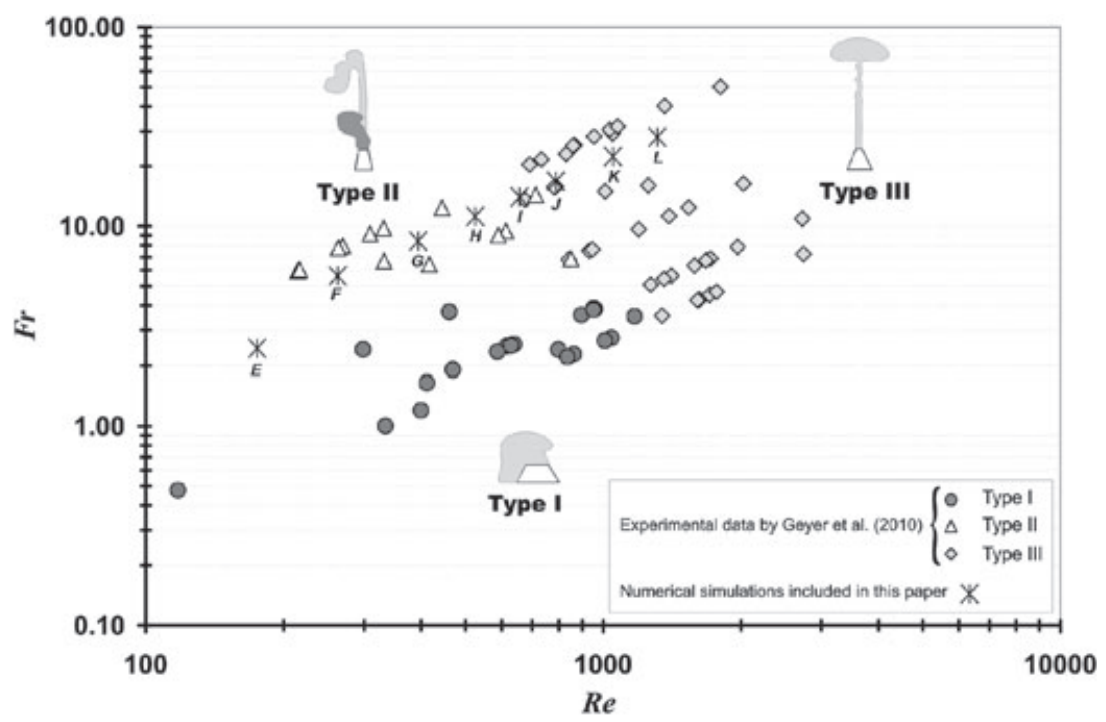

Figure 11. Fr versus $\operatorname{Re}$ plot for the experimental and numerical results presented in this paper. Experimental results are classified according to the flow type: type I, II, or III. 
a)

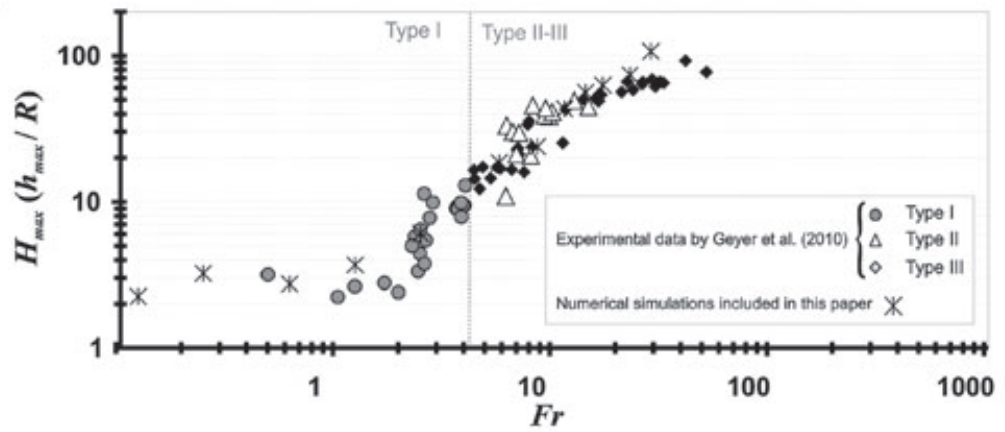

b)

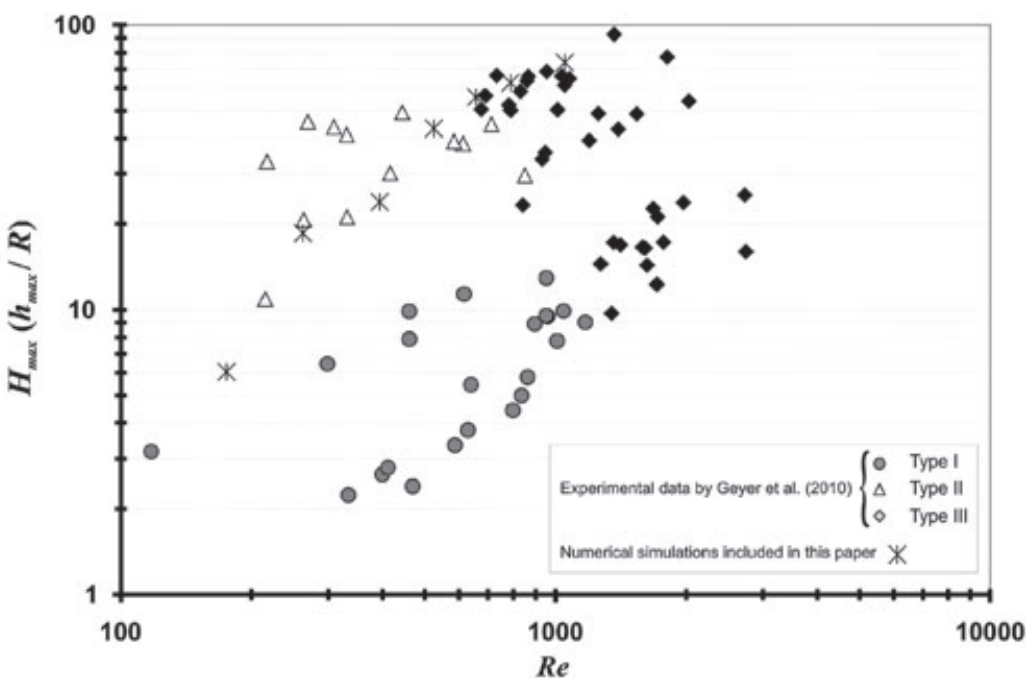

Figure 12. (a) $H_{\max }$ versus $F r$ plot and (b) $H_{\max }$ versus $R e$ plots.

cannot reproduce many aspects of the experiments, but this is a first step in the development of a fully three-dimensional algorithm and allows us to test the capabilities of the method.

\section{SUMMARY AND CONCLUSIONS}

In this paper, we have numerically investigated the dynamics of negatively buoyant jets in a homogenous immiscible fluid using the PFEM. The method is able to model sharp jumps in the physical properties (density and viscosity) of the fluid and take into account the interfacial tension. Interfaces are naturally tracked by the Lagrangian moving mesh, pressure DOFs have been duplicated at the interface nodes to represent the discontinuity of this variable because of surface tension and variable viscosity, surface tension force is applied directly on the interface nodes, and the mesh has been refined in the vicinity of the interface to improve the accuracy and efficiency of the computations.

The simulated process corresponds to the injection of colored water into a cylindrical rapeseed oil tank through a re-entrant nozzle located at the base of the tank. In the different numerical runs, we have varied the injection velocity and the nozzle radius to reproduce a wide range of Froude and Reynolds numbers.

In contrast to previous numerical studies on negatively buoyant jets (Table I) where the density jump between fluids is smoothed by thermal diffusion, viscosity is considered uniform in both fluids and the role of interfacial tension is not taken into account; in the simulations presented here, the participating fluids are immiscible (with realistic physical properties and non-negligible surface tension), and therefore the interface needs to be accurately tracked. Furthermore, the simulations cover 
a larger Froude number interval $(0.1<F r<30)$ and reproduce both weak and strong laminar fountains $(8<R e<1350)$.

The validation of the numerical results with experimental data [26] has shown the potential of the PFEM for studying this kind of challenging flows. We have demonstrated that the method is able to reproduce the main features of all three flow regimes:

1. Type I, characterized by being very stable, that is, the height of the fountain is approximately constant.

2. Type II, described as a pulsating fountain in which height oscillates in time.

3. Type III, observable at higher injection velocities. The jet initially penetrates upward into the ambient fluid, and when it reaches $h_{\max }$, a cap forms at the top.

And additionally, the flow behaviors observed for the different numerical simulations fit in the regime map based on the $R e$ and $F r$ values of the experiments and in the dimensionless maximum fountain height versus $R e$ and $F r$ plots. Numerical and experimental results are in good agreement, suggesting that PFEM is a useful tool for the study of immiscible two-fluid systems.

Simulations on the dynamics of negatively buoyant jets may be applied to several geological situations, for example, subaqueous lava fountains or the replenishment of magma chambers by a dense input of magma. In fact, the results presented in this paper are a first step towards the numerical modeling of three-dimensional magma-ambient fluid interaction (e.g., magma water, dense magma-lighter magma).

\section{ACKNOWLEDGEMENTS}

M. Mier-Torrecilla thanks the Catalan Agency for Administration of University and Research Grants (AGAUR), the European Social Fund, and the CIMNE for their support. A. Geyer is grateful for her postdoctoral Beatriu de Pinos grant 2008 BP B 00318. J.C. Phillips is grateful for the support from the U.S. National Science Foundation (EAR-0810258).

Support from the European Commission and the European Research Council through the 'Real Time Computational Techniques for Multi-Fluid Problems' project is also gratefully acknowledged.

\section{REFERENCES}

1. Campbell IH, Turner JS. Fountains in magma chambers. Journal of Petrology 1989; 30:885-923.

2. Turner JS, Campbell IH. Convection and mixing in magma chambers. Earth-Science Reviews 1986; 23:255-352.

3. Morton BR. Forced plumes. Journal of Fluid Mechanics 1959; 5:151-163. DOI: 10.1017/S002211205900012X.

4. Turner JS. Jets and plumes with negative or reversing buoyancy. Journal of Fluid Mechanics 1966; 26:779-792.

5. Mizushina T, Ogino F, Takeuchi H, Ikawa H. An experimental study of vertical turbulent jet with negative buoyancy. Wärme- und Stoffübertragung 1982; 16:15-21.

6. Bloomfield LJ, Kerr RC. Turbulent fountains in a stratified fluid. Journal of Fluid Mechanics 1998; 358:335-356.

7. Clanet C. On large-amplitude pulsating fountains. Journal of Fluid Mechanics 1998; 366:333-350.

8. Bloomfield LJ, Kerr RC. Turbulent fountains in a confined stratified environment. Journal of Fluid Mechanics 1999; 389:27-54.

9. Bloomfield LJ, Kerr RC. A theoretical model of a turbulent fountain. Journal of Fluid Mechanics 2000; 424:197-216.

10. Philippe P, Raufaste C, Kurowski P, Petitjeans P. Penetration of a negatively buoyant jet in a miscible liquid. Physics of Fluids 2005; 17:17.

11. Kaye NB, Hunt GR. Weak fountains. Journal of Fluid Mechanics 2006; 558:319-328. DOI: 10.1017/S00221120 06000383.

12. Friedman PD. Oscillation in height of a negatively buoyant jet. Journal of Fluids Engineering 2006; 128:880-882.

13. Friedman PD, Meyer WJ, Carey S. Experimental simulation of phase mingling in a subaqueous lava fountain. Journal of Geophysical Research 2006; 111:B07201. DOI: 10.1029/2005JB004162.

14. Friedman PD, Vadakoot DV, Meyer WJ, Carey S. Instability threshold of a negatively buoyant fountain. Experiments in Fluids 2007; 42:751-759. DOI: 10.1007/s00348-007-0283-5.

15. Williamson N, Srinarayana N, Armfield SW, McBain GD, Lin W. Low-Reynolds-number fountain behaviour. Journal of Fluid Mechanics 2008; 608:297-317.

16. Papanicolaou PN, Kokkalis TJ. Vertical buoyancy preserving and non-preserving fountains in a homogeneous calm ambient. International Journal of Heat and Mass Transfer 2008; 51:4109-4120.

17. Lin W, Armfield SW. Very weak fountains in a homogeneous fluid. Numerical Heat Transfer, Part A 2000; 38:377-396.

18. Lin W, Armfield SW. Direct simulation of weak laminar plane fountains in a homogeneous fluid. International Journal of Heat and Mass Transfer 2000; 43:3013-3026. 
19. Lin W, Armfield SW. Direct simulation of weak axisymmetric fountains in a homogeneous fluid. Journal of Fluid Mechanics 2000; 403:67-88.

20. Lin W, Armfield SW. The Reynolds and Prandtl number dependence of weak fountains. Computational Mechanics 2003; 31:379-389.

21. Lin W, Armfield SW. Direct simulation of fountains with intermediate Froude and Reynolds number. ANZIAM Journal 2004; 45:C66-C77.

22. Lin W, Armfield SW. Onset of entrainment in transitional round fountains. International Journal of Heat and Mass Transfer 2008; 51:5226-5237.

23. Srinarayana N, McBain GD, Armfield SW, Lin WX. Height and stability of laminar plane fountains in a homogeneous fluid. International Journal of Heat and Mass Transfer 2008; 51:4717-4727.

24. Williamson N, Armfield SW, Lin W. Direct numerical simulation of turbulent intermediate Froude number fountain flow. ANZIAM Journal 2008; 50:C16-C30.

25. Mier-Torrecilla M, Idelsohn SR, Oñate E. Advances in the simulation of multi-fluid flows with the particle finite element method. Application to bubble dynamics. International Journal for Numerical Methods in Fluids 2010. DOI: 10.1002/fld.2429.

26. Geyer A, Phillips J, Mier-Torrecilla M. Flow behaviour of negatively buoyant jets in immiscible ambient fluid. Experiments in Fluids 2010, submitted.

27. Cresswell RW, Szczepura RT. Experimental investigation into a turbulent jet with negative buoyancy. Physics of Fluids 1993; 5:2865-2878.

28. Idelsohn SR, Oñate E, Calvo N, Pin FD. The meshless finite element method. International Journal for Numerical Methods in Engineering 2003; 58(6):893-912.

29. Idelsohn SR, Calvo N, Oñate E. Polyhedrization of an arbitrary 3D point set. Computer Methods in Applied Mechanics and Engineering 2003; 192:2649-2667. DOI: 10.1016/S0045-7825(03)00298-6.

30. Oñate E, Idelsohn SR, del Pin F, Aubry R. The particle finite element method: an overview. International Journal of Computational Methods 2004; 1(2):267-307.

31. Idelsohn SR, Oñate E, Pin FD. The particle finite element method: a powerful tool to solve incompressible flows with free-surfaces and breaking waves. International Journal for Numerical Methods in Engineering 2004; 61(7):964-989.

32. Oñate E, Idelsohn SR, Celigueta MA, Rossi R. Advances in the particle finite element method for the analysis of fluid-multibody interaction and bed erosion in free surface flows. Computer Methods in Applied Mechanics and Engineering 2008; 197:1777-1800.

33. Larese A, Rossi R, Oñate E, Idelsohn SR. Validation of the particle finite element method (PFEM) for simulation of free surface flows. Engineering Computations 2008; 25:385-425.

34. Idelsohn SR, Oñate E, Pin FD, Calvo N. Fluid-structure interaction using the particle finite element method. Computer Methods in Applied Mechanics and Engineering 2006; 195(17-18):2100-2123.

35. Idelsohn SR, Marti J, Souto-Iglesias A, Oñate E. Interaction between an elastic structure and free-surface flows: experimental versus numerical comparisons using the PFEM. Computational Mechanics 2008; 43:125-132.

36. Idelsohn SR, Pin FD, Rossi R, Oñate E. Fluid-structure interaction problems with strong added-mass effect. International Journal for Numerical Methods in Engineering 2009; 80:1261-1294. DOI: 10.1002/nme.2659.

37. Oñate E, Rossi R, Idelsohn SR, Butler K. Melting and spread of polymers in fire with the particle finite element method. International Journal for Numerical Methods in Engineering 2009; 81:1046-1072. DOI: 10.1002/nme.2731.

38. Idelsohn SR, Mier-Torrecilla M, Nigro N, Oñate E. On the analysis of heterogeneous fluids with jumps in the viscosity using a discontinuous pressure field. Computational Mechanics 2010; 46:115-124. DOI: 10.1007/s00466009-0448-6.

39. Idelsohn SR, Mier-Torrecilla M, Oñate E. Multi-fluid flows with the particle finite element method. Computer Methods in Applied Mechanics and Engineering 2009; 198:2750-2767. DOI: 10.1016/j.cma.2009.04.002.

40. Mier-Torrecilla M. Numerical simulation of multi-fluid flows with the particle finite element method. PhD Thesis, Technical University of Catalonia, 2010. 